\title{
EFEITO DA RADIAÇÃO GAMA SOBRE O PERFIL SENSORIAL DE SUCO DE LARANJA ${ }^{1}$
}

\author{
Marta Regina VERRUMA-BERNARDI ${ }^{2, *}$, Marta Helena F. SPOTO ${ }^{3}$
}

\begin{abstract}
RESUMO
O trabalho teve como objetivo avaliar o efeito de doses de radiações sobre as características sensoriais do suco fresco de laranja variedade 'Pêra' (Citrus sinensis L) conservado sob refrigeração. Utilizou-se amostras de suco de laranja refrigerado (controle) e suco de laranja refrigerado irradiado com 1,5 e 3,0kGy por hora $\left(\right.$ Cobalto ${ }^{60}$ ), armazenadas a $4^{\circ} \mathrm{C}$ por $1,7,14$ e 21 dias a $4^{\circ} \mathrm{C}$. Para a análise sensorial realizou-se a Análise Descritiva Quantitativa, na qual provadores selecionados e treinados avaliaram a aparência, aroma, sabor e textura oral dos sucos. As doses de radiação alteraram características de aparência como cor, brilho e grumosidade. As amostras de suco irradiados apresentaram coloração amarelo mais claro, maior brilho e menor grumosidade que o suco controle. Os sucos irradiados apresentaram também maior intensidade de aroma cozido, passado e artificial, gosto ácido, amargo, sabor cozido, de sumo, passado e de laranja artificial. As doses de radiação utilizadas alteraram as caracteristicas de corpo e adstringência. A cor e a grumosidade do suco não irradiado intensificaram-se com o tempo de armazenamento. Com base nos resultados obtidos, pode-se concluir que a irradiação do suco promoveu alterações nas caracteristicas sensoriais.
\end{abstract}

Palavras-chave: suco de laranja; irradiação; análise descritiva quantitativa.

\section{SUMMARY}

EFFECT OF GAMMA IRRADIATION ON SENSORY PROFILE OF ORANGE JUICE. The objective of this work was to evaluate the characteristics of the irradiated fresh of 'Pera' sweet orange (Citrus sinnensis L.) juice and kept refrigerated. Samples of orange juice (control) and samples irradiated with 1.5 and 3.0kGy per hour (Cobalt ${ }^{60}$ ) were stored for $1,7,14$ and 21 days at $4^{\circ} \mathrm{C}$. Quantitative Descriptive Analysis was conducted by a selected trained panels that evaluated appearance, aroma, flavor and texture of juices. Radiation levels affected appearance characteristics as color, shine and gummy. Irradiated juice resulted more clear and shinny and less gummy, more cooked, with past and artificial aroma, more acid, bitter, cooked, sap, past and with accentuated artificial orange than the control. Irradiation levels affected texture characteristics as body and astringency, homogeneity attribute was not affected. Color and gummy of control sample intensified with the increase of storage periods. Results lead juice irradiation promoted negatively on sensorial characteristic.

Keywords: orange juice; irradiation; quantitative descriptive analysis.

\section{1 - INTRODUÇÃO}

O processo de irradiação consiste na exposição de alimentos a uma fonte de radiação ionizante, minuciosamente controlada, por um tempo pré-fixado. A irradiação impede a divisão de células vivas, tais como bactérias e células de organismos superiores, pois altera suas estruturas moleculares, além de inibir a maturação de alguns vegetais, ao induzir alterações bioquímicas nos processos fisiológicos do tecidos [6].

Nas últimas décadas a irradiação de alimentos tem recebido atenção crescente, devido às vantagens que apresenta em relação aos métodos convencionais de processamento. Dentre as vantagens está a possibilidade de efetuar o tratamento dos alimentos após a embalagem, a conservação das mesmas em no seu estado fresco e possibilitando que em alimentos perecíveis possam ser conservados por mais tempo sem perda da qualidade [11]. As radiações ionizantes, caracterizadas pelas radiações gama, são capazes de esterilizar alimentos e reduzir a carga microbiológica, permitindo assim ampliar o armazenamento [3].

1. Recebido para publicação em 09/08/2000. Aceito para publicação em $17 / 07 / 2002$.

2. Departamento de Nutrição e Dietética - Faculdade de Nutrição / UFF.

Rua São Paulo, $304^{\circ}$ andar-Campus Valonguinho-Centro Niterói - RJ CEP 24110-015E-mail: verruma@bol.com.br

3. Centro de Energia Nuclear na Agricultura - CENA / USP

* A quem a correspondência deve ser enviada.
De acordo com o GCIIA [4], o processo de irradiação acarreta poucas alterações químicas nos alimentos, não sendo conhecidas, nocivas ou perigosas. A variação do valor nutritivo causada pela irradiação depende de vários fatores, entre eles a dose a qual o alimento é exposto, o tipo de alimento, sua embalagem e condições do tratamento, tais como a temperatura durante a irradiação e o tempo de armazenamento. $\mathrm{O}$ tratamento por irradiação se devidamente controlado não altera fisicamente a aparência, a forma ou a temperatura dos produtos, e somente nos alimentos produz alterações químicas insignificantes.

Trabalhos sobre irradiação do suco de laranja, citados por ARTEAGA et al. [2], YÁÑEZ et al. [15], e IEMMA et al. [5] relataram perdas de ácido ascórbico. MUNHOZBURGOS [9] irradiou sucos naturais e concentrados de laranja, tangerina e maracujá, pasteurizados e não pasteurizados, imediatamente enlatados, mostrando que as variações de $\mathrm{pH}$, sólidos solúveis, acidez total e vitamina $\mathrm{C}$ não foram muito significativas até a dose de $0,5 \mathrm{kGy}$, mas com dose de 2,0kGy as variações tornaram significativas.

Estudos descritos por ARTEAGA et al. [2] e YÁÑEZ et al. [15] relataram em que aumentando-se as doses de radiação de 0,25 para $1,5 \mathrm{kGy}$, houve um aumento do $\mathrm{pH}$.

ABDELLAOUI et al. [1] irradiaram tangerinas Clementina com doses entre 0,3 a 0,5kGy de raios gama e observaram a que as características sensoriais não foram afetadas. MITCHELL et al. [7], relataram que doses 
acima de 0,6kGy afetaram negativamente a aceitabilidade do suco com suco de laranja 'Valência'.

De acordo com MUNHOZ-BURGOS [9] o processo combinado de irradiação com aquecimento ou refrigeração, tem sido utilizado na preservação de suco de frutas. No entanto, o seu uso comercial na preservação de sucos de frutas, requer a adequação da dose, a fim de minimizar o aparecimento de características e qualidades sensoriais indesejáveis, e melhorar os aspectos econômicos do processo.

Os efeitos da irradiação de alimentos podem ser determinados em estudos de controle de qualidade, são avaliados os aspectos físicos, químicos e sensoriais ao longo do tempo de prateleira dos alimentos tratados desta forma [11].

De acordo com SPOTO [11] um tratamento promissor para sucos de frutas pode ser a combinação da radiação com refrigeração. Os efeitos da irradiação sobre as características sensoriais das frutas citricas dependem, em grande parte, da dosagem e da temperatura e duração de armazenamento.

Os objetivos deste trabalho foram como avaliar as características sensoriais de suco fresco de laranja irradiado e conservado sob refrigeração.

\section{2 - MATERIAL E MÉTODOS}

\section{1 - Material}

Foi utilizado suco fresco de laranja (Citrus sinensis L), da variedade 'Pêra', as quais foram armazenadas à temperatura entre $22-25^{\circ} \mathrm{C}$ por 72 horas, desde a colheita até o início do processamento. Após lavagem das laranjas e extração, o suco foi envasado em embalagem Tetra Pak ${ }^{\circledR}$. As amostras foram então irradiadas no CENA, com um irradiador Cobalto ${ }^{60}$ da Nordion Canadian, utilizando-se 2 níveis de radiação gama: 1,5 e 3,0kGy e armazenadas a $4^{\circ} \mathrm{C}$ por $1,7,14$ e 21 dias. A amostra controle, foi processada de forma idêntica, sem receber porém nenhum tipo de irradiação ou outro tratamento térmico.

\section{2 - Análise sensorial}

$\mathrm{Na}$ análise sensorial das amostras foi utilizado o Método de Análise Descritiva Quantitativa (ADQ) adaptado da metodologia descrita por STONE \& SIDEL [13].

\subsection{1 - Recrutamento e pré-seleção da equipe}

Dentre os provadores que demonstraram ter interesse e disponibilidade de tempo no período de realização da análise do suco, foram previamente selecionados através de um teste triangular com suco de laranja irradiado e fresco, os indivíduos que obtiveram um mínimo de $60 \%$ de acertos do total de testes realizados. Foi realizado um teste triangular com três repetições.

\subsection{2 - Apresentação das amostras}

As amostras utilizadas na $\mathrm{ADQ}$ foram servidas em copos plásticos à temperatura ambiente, contendo $20 \mathrm{~mL}$, codificados com números de 3 dígitos e apresentadas de forma aleatória. Os testes foram realizados em cabines individuais, utilizando-se luz branca, nos períodos da manhã das 9:00 às 12:00 horas e da tarde das 14:00 às 17:00 horas.

\subsection{3 - Desenvolvimento da terminologia descritiva}

O levantamento da terminologia descritiva das amostras foi feito através do método Rede - "Kelly's Repertory Grid Method" [8]. Foram realizadas 2 sessões e onde foram apresentadas duas amostras de suco (fresco e irradiado), solicitando-se que o provador descrevesse as similaridades e as diferenças entre os sucos, utilizando a Ficha para o levantamento de atributos especificada na Figura 1.

Nome:
Por favor, compare as amostras de suco quanto a aparência, aroma, sabor e textura e descreva
abaixo as similaridades e as diferenças entre os sucos.
Amostras:
Aparência
Aroma
Sabor
Textura
Comentários:

FIGURA 1. Ficha para o levantamento de atributos com o método Rede.

\subsection{4 - Treinamento da equipe}

Após cada provador ter gerado seus termos descritivos para o par de amostras testado, a equipe reuniu-se e sob supervisão de um líder, discutiu os termos levantados. Nesta etapa, os termos que expressavam um mesmo aspecto sensorial foram agrupados em um só atributo, enquanto termos pouco utilizados foram descartados. Ao final de cada sessão, era gerada de forma consensual uma lista de termos descritivos e propostos materiais de referência. Desta maneira, foi gerada uma lista final de termos descritivos, devidamente definidos e com referências de intensidade definidas para os extremos das escalas utilizadas.

$\mathrm{Na}$ fase de treinamento, os provadores foram solicitados a avaliar nas amostras de suco de laranja a intensidade de cada atributo sensorial utilizando uma ficha de avaliação descritiva na qual cada descritor encontrase associado a uma escala estruturada de $10 \mathrm{~cm}$, ancorada à esquerda e à direita nos termos pouco/muito, ou outros termos de intensidade, apresentados na Figura 2. Durante o treinamento, a lista de definições e as referências foram revistas pelos provadores antes das avaliações das amostras. A seleção final dos provadores foi baseada na reprodubilidade dos resultados, poder discriminativo e concordância entre os membros da equi- 
pe. Nesta etapa os nove provadores pré-selecionados permaneceram na equipe.

\subsection{5 - Avaliação das amostra}

As três amostras foram avaliadas por todos os provadores em três repetições. Os provadores utilizaram a ficha de avaliação mostrada na Figura 2. As amostras foram apresentadas aos provadores em ordem aleatorizada.

Nome: Data:
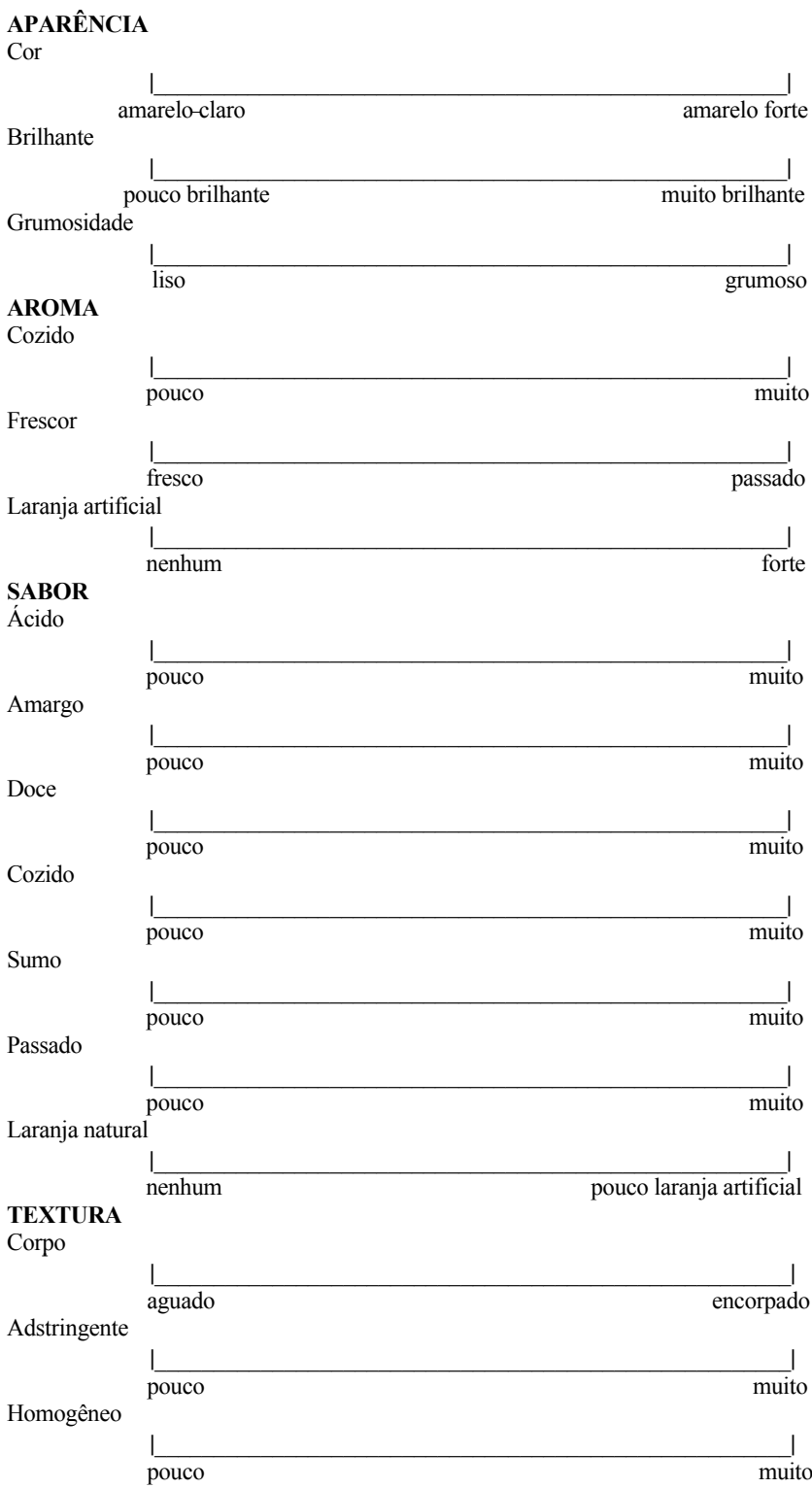

FIGURA 2. Ficha de avaliação utilizada para avaliação das amostras.

\section{3 - Análise estatística}

Os dados obtidos foram avaliados através da análise de variância tendo como fontes de variação: doses e período de armazenamento e foi aplicado o teste de Tukey para a comparação das médias entre as amostras. As análises estatísticas foram realizadas através do programa SAS [10].

\section{3 - RESULTADOS E DISCUSSÃO}

A terminologia descritiva das amostras inclui 3 atributos para aparência (cor, brilho e grumosidade), 3 para aroma (cozido, frescor, laranja artificial), 7 para sabor (ácido, amargo, doce, cozido, sumo, passado e laranja natural) e 3 para textura (corpo, adstringência e homogêneo), cujas definições encontram-se apresentadas na Tabela 1.

SPOTO [12] estudando o perfil sensorial de sabor do suco de laranja concentrado irradiado, obteve os seguintes atributos descritivos: laranja, ácido, remédio, cozido, óleo e doce.

TABELA 1. Definição dos atributos sensoriais avaliados.

\begin{tabular}{|c|c|}
\hline \multicolumn{2}{|r|}{ APARÊENCIA } \\
\hline Cor & Amarelo claro: refere-se a cor amarela clara. \\
\hline & Amarelo escuro: refere-se a cor amarelo ouro. \\
\hline Brilhante & $\begin{array}{l}\text { Pouco: refere-se suco diluído em água sem brilho. } \\
\text { Muito: refere-se a um alimento que brilha. }\end{array}$ \\
\hline Grumosidade & $\begin{array}{l}\text { Liso: refere-se que suco que possui superfície plana, lisa., sem } \\
\text { aspereza. } \\
\text { Grumoso: refere-se ao suco com grumos. }\end{array}$ \\
\hline \multicolumn{2}{|r|}{ AROMA } \\
\hline Cozido & Refere-se ao aroma da laranja cozida. \\
\hline Frescor & Fresco: refere-se a fruta recentemente colhida. \\
\hline Laranja artificial & $\begin{array}{l}\text { Artificial: refere-se ao suco aromatizado artificialmente como pó } \\
\text { para refresco de laranja. }\end{array}$ \\
\hline \multicolumn{2}{|r|}{ SABOR } \\
\hline Ácido & $\begin{array}{l}\text { Refere-se ao gosto característico de água mais ácido cítrico em } \\
\text { solução aquosa. }\end{array}$ \\
\hline Amargo & $\begin{array}{l}\text { Refere-se ao gosto característico de quinino/cafeína em solução } \\
\text { aquosa. }\end{array}$ \\
\hline Doce & Refere-se ao gosto característico de sacarose em solução aquosa. \\
\hline Cozido & Refere-se ao sabor de laranja cozida. \\
\hline Sumo & Refere-se ao líquido que sai no momento de descascar a laranja. \\
\hline Passado & Refere-se à laranja quando começa apodrecer. \\
\hline Laranja natural & Natural: refere-se ao sabor da fruta in natura. \\
\hline \multicolumn{2}{|r|}{ TEXTURA } \\
\hline Corpo & $\begin{array}{l}\text { Aguado: refere-se a fruta que contém muita água e pouco açúcar. } \\
\text { Encorpado: forte, consistente. Ex. vitamina de frutas. }\end{array}$ \\
\hline Adstringência & $\begin{array}{l}\text { Refere-se a propriedade de um alimento "amarrar" a boca. Ex. } \\
\text { banana-maçã verde, caqui verde. }\end{array}$ \\
\hline Homogêneo & $\begin{array}{l}\text { Refere-se ao suco que contêm elementos idênticos por toda sua } \\
\text { natureza. }\end{array}$ \\
\hline
\end{tabular}

De acordo com os resultados obtidos (Tabela 2), pode-se verificar que as doses de radiação (1,5 e 3,0kGy) modificaram as características de aparência de cor, brilho e grumosidade. As amostras dos sucos irradiados apresentaram uma coloração amarelo mais claro, maior brilho e menor grumosidade que o suco controle. Com relação ao período de armazenamento, a cor e a grumosidade intensificaram-se apenas no caso da amostra controle. SPOTO [12], estudando o efeito do armazenamento de suco de laranja concentrado concluiu que a temperatura de armazenamento prejudica a qualidade do suco.

De acordo com os resultados apresentados na Tabela 3, verifica-se que as amostras dos sucos irradiados apresentaram menor intensidade para o atributo frescor e maior intensidade para o aroma cozido e laranja artificial, enquanto a amostra controle apresentou no- 
tas bem baixas para estes atributos, indicando que a irradiação, nas doses utilizadas induziram alterações sensoriais com relação ao aroma do suco de laranja fresco e refrigerado. Verificou-se que com relação a período de armazenamento ocorreram diferenças nas notas atribuidas pelos provadores para todos os atributos de aroma cozido, fresco e aroma laranja artificial.

TABELA 2. Efeito das doses de radiação e períodos (dias) de armazenamento nos atributos de aparência ${ }^{1,2}$.

\begin{tabular}{cccccccccr}
\hline & & Cor & \multicolumn{3}{c}{ Brilho } & \multicolumn{3}{c}{ Grumosidade } \\
\hline & \multicolumn{1}{c}{ Doses (kGy) } & & & \\
\hline Dias & $\mathbf{0 , 0}$ & $\mathbf{1 , 5}$ & $\mathbf{3 , 0}$ & $\mathbf{0 , 0}$ & $\mathbf{1 , 5}$ & $\mathbf{3 , 0}$ & $\mathbf{0 , 0}$ & $\mathbf{1 , 5}$ & $\mathbf{3 , 0}$ \\
\hline $\mathbf{1}$ & $5,47 \mathrm{aA}$ & $5,86 \mathrm{aA}$ & $5,54 \mathrm{aA}$ & $6,65 \mathrm{aA}$ & $6,31 \mathrm{aA}$ & $6,37 \mathrm{aA}$ & $2,14 \mathrm{cB}$ & $5,13 \mathrm{aA}$ & $4,04 \mathrm{aB}$ \\
$\mathbf{7}$ & $8,83 \mathrm{aA}$ & $3,44 \mathrm{abB}$ & $3,72 \mathrm{aB}$ & $4,76 \mathrm{aA}$ & $6,40 \mathrm{aA}$ & $6,17 \mathrm{aA}$ & $5,67 \mathrm{bA}$ & $2,30 \mathrm{aB}$ & $2,83 \mathrm{aB}$ \\
$\mathbf{1 4}$ & $8,11 \mathrm{aA}$ & $5,15 \mathrm{bB}$ & $4,71 \mathrm{aB}$ & $5,08 \mathrm{aB}$ & $7,40 \mathrm{aA}$ & $7,15 \mathrm{aA}$ & $7,77 \mathrm{aA}$ & $3,05 \mathrm{aB}$ & $3,41 \mathrm{aB}$ \\
$\mathbf{2 1}$ & $8,20 \mathrm{aA}$ & $5,08 \mathrm{abB}$ & $4,73 \mathrm{aB}$ & $3,97 \mathrm{aB}$ & $7,01 \mathrm{aA}$ & $6,85 \mathrm{aA}$ & $8,95 \mathrm{aA}$ & $3,44 \mathrm{aB}$ & $2,42 \mathrm{aB}$ \\
\hline
\end{tabular}

${ }^{1}$. Na vertical, médias com letras (minúsculas) em comum não diferem entre si a $5 \%$ significância.

2 . Na horizontal, médias com letras (maiúsculas) em comum não diferem entre si a $5 \%$ significância.

TABELA 3. Efeito das doses de radiação e períodos (dias) de armazenamento nos atributos de aroma ${ }^{1,2}$.

\begin{tabular}{cccccccccc}
\hline & & Cozido & \multicolumn{3}{c}{ Frescor } & \multicolumn{3}{c}{ Laranja artificial } \\
\hline & \multicolumn{9}{c}{ Doses (kGy) } \\
\hline Dias & $\mathbf{0 , 0}$ & $\mathbf{1 , 5}$ & $\mathbf{3 , 0}$ & $\mathbf{0 , 0}$ & $\mathbf{1 , 5}$ & $\mathbf{3 , 0}$ & $\mathbf{0 , 0}$ & $\mathbf{1 , 5}$ & $\mathbf{3 , 0}$ \\
\hline $\mathbf{1}$ & $0,62 \mathrm{aB}$ & $7,21 \mathrm{aA}$ & $4,48 \mathrm{aA}$ & $0,91 \mathrm{aB}$ & $6,53 \mathrm{aA}$ & $4,74 \mathrm{aA}$ & $1,51 \mathrm{aB}$ & $6,26 \mathrm{aA}$ & $4,70 \mathrm{aA}$ \\
$\mathbf{7}$ & $2,24 \mathrm{aB}$ & $6,57 \mathrm{aA}$ & $5,22 \mathrm{aA}$ & $2,54 \mathrm{aB}$ & $6,52 \mathrm{aA}$ & $5,18 \mathrm{aA}$ & $2,64 \mathrm{aB}$ & $6,63 \mathrm{aA}$ & $5,32 \mathrm{aA}$ \\
$\mathbf{1 4}$ & $0,64 \mathrm{aB}$ & $6,84 \mathrm{aA}$ & $5,01 \mathrm{aA}$ & $2,45 \mathrm{aB}$ & $6,74 \mathrm{aA}$ & $5,14 \mathrm{aA}$ & $0,94 \mathrm{aB}$ & $7,60 \mathrm{aA}$ & $5,51 \mathrm{aA}$ \\
$\mathbf{2 1}$ & $0,81 \mathrm{aB}$ & $7,04 \mathrm{aA}$ & $4,97 \mathrm{aA}$ & $2,25 \mathrm{aB}$ & $6,55 \mathrm{aA}$ & $4,99 \mathrm{aA}$ & $0,66 \mathrm{aB}$ & $7,00 \mathrm{aA}$ & $5,02 \mathrm{aA}$ \\
\hline
\end{tabular}

${ }^{1}$. Na vertical, médias com letras (minúsculas) em comum não diferem entre si a $5 \%$ significância.

. Na horizontal, médias com letras (maiúsculas) em comum não diferem entre si a $5 \%$ significância.

Para os atributos de sabor, os sucos irradiados apresentaram gosto mais ácido à medida que aumentou a dose de radiação. As amostras que irradiadas também apresentaram sabor cozido, sumo e passado mais acentuados que a amostra controle. O atributo sabor laranja foi mais intenso para a amostra controle e no caso do sabor doce, os provadores não perceberam diferenças entre as amostras.

Pode-se verificar na Tabela 4, para os atributos sabor passado e cozido, pode-se dizer que os provadores detectaram um aumento progressivo deste atributo nas amostras radiadas a medida que aumentavam os dias de armazenamento. O mesmo não ocorreu na amostra controle. VELEZ et al. [14] relataram que o armazenamento a $0^{\circ} \mathrm{C}$ e durante curto tempo a $20^{\circ} \mathrm{C}$ raramente afeta o aroma do suco de laranja, porém em longos períodos a $20^{\circ} \mathrm{C}$ pode afetar.

Estudos descritos MUNHOZ-BURGOS [9] mostraram que a radiação gama em suco de laranja modifica as suas características sensoriais sendo que os principais atributos afetados até uma dose de 1,0kGy são cor, aroma e sabor. MITCHELL et al. [7], relataram que doses acima de 0,6kGy afetaram negativamente a aceitabilidade do suco com suco de laranja 'Valência'.

Para os atributos relativos a textura (Tabela 5), o atributo corpo da amostra controle, aumentou depois do primeiro dia. Este resultado provavelmente está relacionado com a aparência de grumosidade acentuada, também relatada pelos provadores. As doses de irradiação também modificaram a adstringência das amostras, diferenciando as amostras irradiadas.

TABELA 4. Efeito das doses de radiação e períodos (dias) de armazenamento nos atributos de sabor ${ }^{1,2}$.

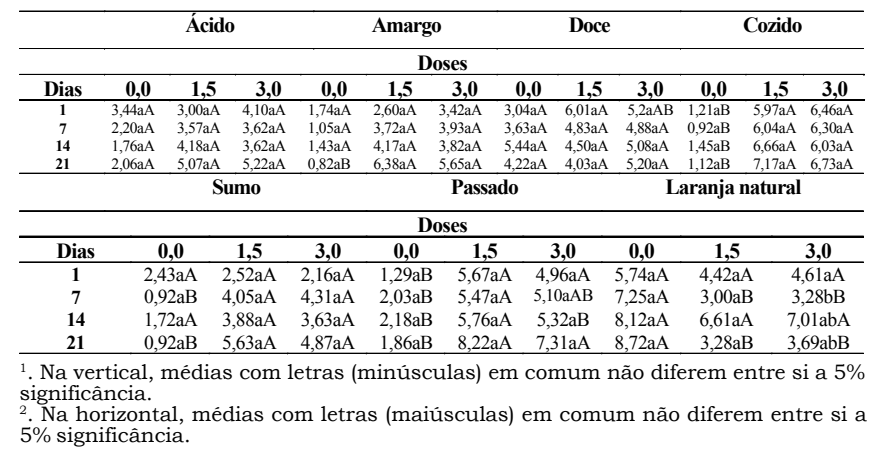

TABELA 5. Efeito das doses de radiação e períodos (dias) de armazenamento nos atributos de textura ${ }^{1,2}$.

\begin{tabular}{lccccccccc}
\hline & \multicolumn{1}{c}{ Corpo } & \multicolumn{4}{c}{ Adstringente } & \multicolumn{2}{c}{ Homogêneo } \\
\hline \multicolumn{1}{c}{ Doses } & $\mathbf{0}$ & & & \\
\hline Dias & $\mathbf{0 , 0}$ & $\mathbf{1 , 5}$ & $\mathbf{3 , 0}$ & $\mathbf{0 , 0}$ & $\mathbf{1 , 5}$ & $\mathbf{3 , 0}$ & $\mathbf{0 , 0}$ & $\mathbf{1 , 5}$ & $\mathbf{3 , 0}$ \\
\hline $\mathbf{1}$ & $5,06 \mathrm{bA}$ & $6,76 \mathrm{aA}$ & $7,35 \mathrm{aA}$ & $1,98 \mathrm{aA}$ & $1,97 \mathrm{aA}$ & $2,06 \mathrm{aA}$ & $5,25 \mathrm{aA}$ & $4,20 \mathrm{aA}$ & $3,93 \mathrm{aA}$ \\
$\mathbf{7}$ & $7,91 \mathrm{abA}$ & $6,74 \mathrm{aA}$ & $6,74 \mathrm{aA}$ & $0,76 \mathrm{aA}$ & $2,22 \mathrm{aA}$ & $2,08 \mathrm{aA}$ & $5,30 \mathrm{aA}$ & $5,82 \mathrm{aA}$ & $6,28 \mathrm{aA}$ \\
$\mathbf{1 4}$ & $8,41 \mathrm{aA}$ & $6,96 \mathrm{aA}$ & $7,10 \mathrm{Aa}$ & $0,80 \mathrm{aA}$ & $2,86 \mathrm{aA}$ & $2,77 \mathrm{aA}$ & $3,77 \mathrm{aA}$ & $5,78 \mathrm{aA}$ & $6,13 \mathrm{aA}$ \\
$\mathbf{2 1}$ & $7,43 \mathrm{abA}$ & $5,96 \mathrm{aA}$ & $5,94 \mathrm{aA}$ & $1,18 \mathrm{aB}$ & $5,21 \mathrm{aA}$ & $4,09 \mathrm{aAB}$ & $3,87 \mathrm{aA}$ & $5,65 \mathrm{aA}$ & $5,40 \mathrm{aA}$ \\
\hline 1. Na vertical, médias com letras (minúsculas) em comum não diferem entre si a 5\% \\
significância. \\
2. Na horizontal, médias com letras (maiúsculas) em comum não diferem entre si a \\
5\% significância.
\end{tabular}

\section{4 - CONCLUSÕES}

- o uso da Análise Descritiva Quantitativa, possibilitou o levantamento de atributos sensoriais importantes para a discriminação das amostras do suco de laranja fresco e refrigerado em comparação das amostras de suco submetidos à radiação.

- os resultados mostraram que as doses de radiação utilizadas afetaram as características de aparência, aroma, sabor e textura.

- Com base nos resultados obtidos pode-se afirmar que a irradiação promoveu alterações de características sensoriais que provavelmente terão influência negativa na aceitabilidade do suco, assim como afetarão sua qualidade.

\section{5 - REFERÊNCIAS}

[1] ABDELLAOUI, S.; LACROIX, M.; JOBIN, M.; BOUBEKRI, C.; GAGNON, M.; AIT-OUTBAHOU, A; EL-OTMANI, M. Efet de i'irradiation gamma avec et sans traitement a i'eau chaud sur les qualites physico-chimique, nutritionnelles et organoleptiques des clementines. In: Postharvest physiology, pathology and technologies for horticultural commodities: recent advances. Proceedings... Agadir, 1994.p.436-445.

[2] ARTEAGA, A.G.; YÁÑEZ, M.G.; MIRANDA, J.F.; PARAODA, A.; SAMPERE, E.; SERRANO, G. Estalidad del contenido de vitamina $\mathrm{C}$ e algunos indicadores químicos en naranjas sometidas a diferentes dosis de radiaciones gamma. Revista Alimentaria, v.198, p.45-46, 1988.

[3] FIGUEIREDO, F. Irradiação de alimentos. Alimentos \& Tecnologia. v.6, n.30, p.96-98, 1990. 
[4] GRUPO CONSULTIVO INTERNACIONAL SOBRE IRRADIAÇÃO DE ALIMENTOS - GCIIA. A irradiação de alimentos: ficção e realidade. Ficha Descritiva 1-14. 1990.

[5] IEMMA, J.; ALCARDE, A R.; DOMARCO, R.E.; SPOTO, M.H.F.; BLUMER, L.; MATRAIA, C. Radiação gama na conservação do suco de laranja natural. Scientia Agricola, v.56, n.4, p.1193-1198, 1999.

[6] INTERNATIONAL ATOMIC ENERGY AGENGY - IAEA. Facts about food irradiation. Vienna, 1991. 38p. (IAEA / PI / A33E).

[7] MITCHELL, G.E.; ISAACS, A. R.; WILliAMS, D.J.; McLAUCHLAN, R.L.; NOTTINGHAM, S.M.; HAMMETON. $\mathrm{K}$. Low dose irradiation influence on yield and quality of fruit juice. Journal of Food Science. v. 56, n. 6, p. 16281631, 1991.

[8] MOSKOWITZ, H.R. Product testing and sensory evalution of foods. Westport: Food \& Nutrition Press, 1983. 605p.

[9] MUNHOZ-BURGOS, R.A. Uso de la radiacion gamma para extender el tiempo de conservation de jugos de algunas frutas exóticas. In: Seminário sobre Irradiação de Alimentos para Países da América Latina, Quito, 1985. Actas. Vienna, IAEA, p.1-25, 1985.
[10] SAS INSTITUTE INC. SAS/STAT; user's guide: version 6, 4. ed. Cary, SA, 1989. v.2, 846p.

[11] SPOTO, M.H.F. Radiação gama na conservação de suco concentrado de laranja: características físicas, químicas e sensoriais. Piracicaba, 1988.91p. Tese de Mestrado - ESALQ/USP).

[12] SPOTO, M.H.F. Os componentes químicos relacionados ao sabor e aroma do suco de laranja concentrado e irradiado. Piracicaba, 1993. 127p. Tese de Doutorado-ESALQ/ USP.

[13] STONE, H.S; SIDEL, J.L. Sensory evalution practices. London: Academic Press, 1985. 311p.

[14] VELEZ, C.; COSTELL, E.; ORLANDO, L.; NADAL, M.I.; SENDRA, J.M.; IZQUIERDO, L. Multidimensional scaling as method to correlate sensory and instrumental data of orange juices aromas. Journal Science and Food Agriculture. v.61, p.41-46, 1993.

[15] YÁÑES, M.G. ARTEAGA, A.G.; YÁÑEZ, M.G.; MIRANDA, J.F.; PARAODA, A.; SAMPERE, E.; SERRANO, G. Nota: Estalidad del contenido de vitamina $\mathrm{C}$ en torontas sometidas a diferentes dosis de radiaciones gamma. Revista de Agroquimica e Tecnologia de Alimentos, v.30, n.3, p.409-416, 1990. 